\title{
Mapping between the Heisenberg XX Spin Chain and Low-Energy QCD
}

\author{
David Pérez-García and Miguel Tierz \\ Departamento de Análisis Matemático and Instituto de Matemática Interdisciplinar, \\ Universidad Complutense de Madrid, 28040 Madrid, Spain \\ (Received 14 August 2013; revised manuscript received 5 March 2014; published 16 June 2014) \\ By using random matrix models, we uncover a connection between the low-energy sector of \\ four-dimensional QCD at finite volume and the Heisenberg XX model in a 1D spin chain. This connection \\ allows us to relate crucial properties of QCD with physically meaningful properties of the spin chain, \\ establishing a dictionary between both worlds. For the spin chain, we predict a third-order phase transition \\ and a Tracy-Widom law in the transition region. We also comment on possible numerical implications of \\ the connection as well as on possible experimental implementations.
}

DOI: 10.1103/PhysRevX.4.021050

\section{INTRODUCTION}

The strong interaction is the fundamental force of nature that describes the interaction between quarks and gluons, the elementary constituents of hadronic matter. It is described by QCD, a $S U(3)$ Yang-Mills theory with a number of distinctive properties, such as asymptotic freedom [1], which correctly describes that the interaction between particles becomes asymptotically weaker as distance decreases and energy increases. This crucial property, in agreement with Bjorken scaling and experimental data, is due to the negativity of the $\beta$ function describing the variation of the coupling constant of the theory under the renormalization group flow [1]. Phenomenology also tells us that the up and down quarks are very light. The case of massless quarks implies some additional symmetries, named chiral symmetries, which would allow separate transformations between the left-handed quarks and the right-handed ones. Such behavior is not observed and, hence, in a realistic QCD, the chiral symmetry must be spontaneously broken. Low-energy QCD, which is the regime we are interested in, is deeply related to the notion of chiral symmetry breaking and it can be explored with chiral perturbation theory [2-4]. Recall that quarks interact weakly at high energies and strongly at low energies and, therefore, the low-energy regime is described by nonperturbative physics. Finally, chief among the features of QCD is the confinement of quarks into hadrons, either mesons $(q \bar{q})$ or baryons $(q q q)$. Confinement in a gauge theory is usually probed by studying the behavior of Wilson loops observables [5].

Published by the American Physical Society under the terms of the Creative Commons Attribution 3.0 License. Further distribution of this work must maintain attribution to the author(s) and the published article's title, journal citation, and DOI. Subject Areas: Condensed Matter Physics,
Interdisciplinary Physics,

Particles and Fields

There have been a lot of theoretical and numerical approaches to analyzing QCD and related gauge theories, such as effective field theory and chiral perturbation theory $[2,3]$, lattice gauge theory [6,7], the light-cone quantization [8], gauge-string duality, and AdS/CFT approaches [9], etc. Very recently, and motivated by an idea of Feynman [10], a new route has appeared to understand Abelian and nonAbelian gauge theory: simulating it in a different controllable quantum system, such as cold atoms in optical lattices $[11,12]$. Some first steps for different quantum field theories have been carried out in Refs. [13-20].

In this paper, we initiate a different, but somehow related, approach. By combining a result of Leutwyler and Smilga [21] (based on the previous seminal work on chiral perturbation theory $[3,4]$ ) with a result of Bogoliubov et al. [22-25], we uncover a mapping between the lowenergy sector of QCD with thermal correlation functions in the 1D Heisenberg XX model or, via a Jordan-Wigner transformation [26], thermal correlation functions in a 1D free-fermion system. The connection is made by relating both objects to a random matrix model [27]. Building upon this starting point, we are then able to relate crucial properties of QCD with physically meaningful properties of the spin chain. For instance, we show that (1) the number of flavors in QCD corresponds to the number of particles (spins down) in the 1D chain, (2) the topological charge in QCD is associated with the signature that topological 2D systems leave on their boundary theory [28-31], (3) different matter content, such as Majorana fermions, corresponds to different boundary conditions in the spin chain, or (4) putting QCD on the lattice enforces the addition of next-to-nearest neighbor terms in the spin chain Hamiltonian. The connection allows us to also uncover a third-order phase transition in the XX model since, again via random matrix models, one can relate both low-energy QCD and the thermal correlation functions of the XX chain 
TABLE I. Dictionary relating properties of QCD with properties of the thermal averages in the XX spin chain.

\begin{tabular}{ll}
\hline \hline Low-energy QCD & Thermal correlations (XX model) \\
\hline Number of flavors & Number of particles \\
Topological sector & Ket versus bra shift \\
$\theta$ angle & Projection onto momentum $\theta$ \\
Different matter content & Different boundary conditions \\
On the lattice & Longer range interactions \\
\hline \hline
\end{tabular}

with the so-called Gross-Witten model, a 2D Yang-Mills theory with gauge group $U(N)$ and no matter fields, which has a third-order phase transition in the limit $N \rightarrow \infty$ [32]. Finally, the connection opens the possibility of using numerical methods coming from spin chains [33] to give good estimates for the partition function of lowenergy QCD. It also opens a way to measure this partition function or to observe the Gross-Witten phase transition experimentally.

\section{LOW-ENERGY QCD AS A RANDOM MATRIX MODEL}

Let us start by describing the derivation in Ref. [21], which applies the ideas of effective field theory [2] to the study of the meson sector of QCD [4,21]. Recall that the main idea of an effective field theory approach is to integrate out the heavy degrees of freedom (the most massive fields) of the theory. This is implemented to study the low-energy (meson) sector of QCD, through chiral perturbation theory [3] with the quark and gluon fields of QCD replaced by a set of pion fields $U(x)$, which describe the degrees of freedom of the pseudo-Nambu-Goldstone bosons. The effective Lagrangian depends only on the pion fields and its derivatives:

$$
\mathcal{L} \rightarrow \mathcal{L}_{\text {eff }}(U, \partial U, \ldots)=\mathcal{L}_{\text {eff }}^{(0)}+\mathcal{L}_{\text {eff }}^{(2)}+\mathcal{L}_{\text {eff }}^{(4)}+\cdots
$$

Chiral symmetry provides a tight constraint to the form of these terms and, in particular, the first term $\mathcal{L}_{\text {eff }}^{(0)}$ is just a constant that is the vacuum energy of QCD in the chiral limit. The first nontrivial term is $\mathcal{L}_{\text {eff }}^{(2)}$ and is given by [2,3]

$$
\mathcal{L}_{\text {eff }}^{(2)}=\frac{1}{4} F^{2} \operatorname{tr}\left\{\partial_{\mu} U^{\dagger} \partial^{\mu} U\right\}+\frac{1}{2} F^{2} \Sigma \operatorname{tr}\left\{M\left(U+U^{\dagger}\right)\right\},
$$

where $M$ is the matrix that contains the masses of the quarks (quark mass matrix) and that will be taken below to be a multiple of the identity matrix, $F$ is the pion decay constant, and $\Sigma$ is the chiral condensate (which describes the spontaneous chiral symmetry breaking).

Chiral perturbation theory gives an expansion in powers of $M$, temperature $T$, and inverse length $1 / L$ at fixed $\Lambda_{\mathrm{QCD}}$ and at fixed ratios $M / T^{2}$ and $L T$. The scales $T$ and $1 / L$ are treated as small quantities of order $p$, where $p$ is the momentum of the pions, whereas the quark mass matrix counts as a quantity of order $p^{2}$ [4]. The most general gauge-invariant expression consistent with the symmetries of QCD that can be formed within the effective theory at $\mathcal{O}\left(p^{2}\right)$ is given by Eq. (1). This Lagrangian holds under the condition for the volume $V^{1 / 4} \gg 1 / \Lambda_{\mathrm{QCD}}$, where $\Lambda_{\mathrm{QCD}}$ is the length scale of QCD [4]. In this way, only the Goldstone modes contribute to the mass dependence of the partition function [4].

In addition, for quark masses for which the Compton wavelength of the Goldstone modes is much larger than the size of the box, $1 / m_{\pi} \gg V^{1 / 4}$ [34]. This is known as the epsilon regime of QCD since it is an expansion in terms of $\varepsilon^{2} \sim\left(m_{\pi} / \Lambda_{\mathrm{QCD}}\right)$ [4]. The fluctuations of the zeromomentum modes of the pion fields dominate the fluctuations of the nonzero momentum modes, and only the former are taken into account in the thermal average [21]. These two conditions on the volume are also referred to as the kinetic domain [35] since the kinetic term of the chiral Lagrangian can be ignored. The low-energy partition function is then [4]

$$
Z_{\mathrm{eff}}(M, \theta)=\int_{U \in S U\left(N_{f}\right)} d U \exp \left(V \Sigma \operatorname{Re} \operatorname{tr}\left\{M U^{\dagger}\right\} e^{i \theta / N_{f}}\right)
$$

since only the constant fields contribute to its mass dependence.

Note that the inverse temperature $\beta$ of the gauge theory does not appear since, in the low-energy effective field theory, one can absorb it in the low-energy constants [4]. The appearance of the $\theta$ parameter is because, due to the explicit breaking of the axial symmetry $U_{A}(1)$, one is naturally led to also consider the addition of a theta term to the original QCD Lagrangian [21],

$$
\mathcal{L}_{\theta}=-\frac{i \theta}{32 \pi^{2}} F_{\mu \nu}^{a} \tilde{F}_{\mu \nu}^{a}
$$

where the field strength and its dual are given by [36]

$$
F_{\mu \nu}^{a}=\partial_{\mu} A_{\nu}^{a}-\partial_{\nu} A_{\mu}^{a}+f_{a b c} A_{\mu}^{b} A_{\nu}^{c}, \quad \tilde{F}_{\mu \nu}^{a}=\frac{1}{2} \varepsilon_{\mu \nu \alpha \beta} F^{\alpha \beta},
$$

with $f_{a b c}$ the structure constants of the gauge group $S U\left(N_{c}\right)$. This is the same topological term that appears in topological insulators [37]. The topological charge

$$
\nu=\frac{1}{32 \pi^{2}} \int F_{\mu \nu}^{a} \tilde{F}_{\mu \nu}^{a} d^{4} x
$$

characterized by the integer $\nu$, is a topological invariant. The low-energy QCD partition function is then written as [21] 


$$
Z_{\mathrm{QCD}}^{\mathrm{eff}}(M, \theta)=\sum_{\nu=-\infty}^{\infty} e^{i \nu \theta} Z_{\nu}^{\mathrm{eff}}(M)
$$

The effective partition function at fixed $\nu$ then follows from Fourier inversion [21]

$$
Z_{\nu, N_{f}}^{\mathrm{eff}}(M)=\int_{U\left(N_{f}\right)} d U[\operatorname{det}(U)]^{\nu} \exp \left(\frac{V \Sigma}{2} \operatorname{Tr}\left[\left(M\left(U+U^{\dagger}\right)\right]\right) .\right.
$$

Now, using Weyl's integration formula, and assuming that $M$ is a multiple of the identity, that is, the masses of all quarks are taken identically $m$, one obtains [21]

$$
\begin{aligned}
Z_{\nu, N_{f}}^{\mathrm{eff}}(m)= & \frac{1}{(2 \pi)^{N_{f}} N_{f} !} \int_{-\pi}^{\pi} d \varphi_{1} \ldots \\
& \times \int_{-\pi}^{\pi} d \varphi_{N_{f}} \prod_{1 \leq j<k \leq N_{f}}\left|e^{i \varphi_{k}}-e^{i \varphi_{j}}\right|^{2} \\
& \times\left(\prod_{j=1}^{N_{f}} e^{V \Sigma m \cos \left(\varphi_{j}\right)}\right)\left(\prod_{j=1}^{N_{f}} e^{i \nu \varphi_{j}}\right) .
\end{aligned}
$$

Notice that this matrix model [an integral of this type with a Vandermonde term $\left|e^{i \varphi_{k}}-e^{i \varphi_{j}}\right|^{2}=4 \sin ^{2}\left(\varphi_{k}-\varphi_{j} / 2\right)$ is a unitary random matrix ensemble [27]], in the case $\nu=0$, is the Gross-Witten matrix model that appeared in the study of lattice 2D Yang-Mills theory with the Wilson lattice action $[32,38]$. In that theory, there is no matter, so it corresponds to two-dimensional gluodynamics. Notice, however, a crucial difference between the appearance of the matrix model in the two theories: in the 2D Yang-Mills theory, $N_{f}=0$ and the group integration is then over $U(N)$, which corresponds to $U\left(N_{c}\right)$ in the $4 \mathrm{D}$ QCD case. Note that, as pointed out in Ref. [35], the matrix integration (3) is over $U\left(N_{f}\right)$, the flavor space, and, hence, while the model is identical, the description is very different. Taking this into account, we also focus on the very distinctive property of the Gross-Witten matrix model [32]: a third-order phase transition in the $N \rightarrow \infty$ limit, which has been the object of intense interest over three decades, since it plays a rather paradigmatic role in the study of confinement and deconfinement and Hagedorn phase transitions [39] and has also been a guide in the study of phase transitions in 4D Yang-Mills theory [40]. We thus discuss both aspects of the correspondence with the spin chain: the description of low-energy QCD in terms of the spin chain and the implications of the Gross-Witten phase transition on the spin chain model.

\section{A. Complexity of the Leutwyler-Smilga integral}

Before proceeding to establishing and exploiting a spin chain representation of Eqs. (2) and (4), we discuss some aspects of their numerical evaluation. In particular, by calling $\beta=V \Sigma m$, Eq. (4) is an integral representation of $\operatorname{det}\left[I_{i-j+\nu}(\beta)\right]_{i, j}^{n}$, where $I_{\nu}(\beta)$ denotes the modified Bessel function of second order, which is the $\nu$ th Fourier coefficient of the weight function of the matrix model, namely, $e^{\beta \cos \theta}$.

The numerical evaluation of the Bessel function for a fixed small value of the order $\nu$ is immediate since a numerical evaluation of its integral representation with the trapezoidal rule is exponentially convergent [41].

However, its evaluation for large values of the order $\nu$ and the argument $\beta$ is a notoriously complex problem, and the development of numerical implementations of uniform asymptotic expansions of the Bessel function in that regime is the subject of much current interest (see Ref. [42] and references therein). Indeed, even though the problem of its evaluation goes back to Debye [43], who devised nonuniform asymptotic expansions, and the fact that nontrivial uniform asymptotic expansions were found in the 1950s $[44,45]$, it turns out that the coefficients of the asymptotic expansion not only exhibit resurgence [46] but also involve the evaluation of higher transcendental functions, in this case, Airy functions.

More specifically, the direct application of the uniform asymptotics becomes problematic when the argument and the order of a Bessel function are almost equal, due to huge numerical cancellations involved in evaluating the individual coefficients in the uniform asymptotic expansions [42] (which is a consequence of the confluence of two saddles in the steepest-descent study of the integral representation of the function).

The phenomena of the appearance and coalescence of saddles is, of course, specially relevant when $\beta$ is complex, due to the different ensuing crossings of Stokes lines in the steepest-descent study of the integral representation of the Bessel function [47].

At any rate, the numerical evaluation of Eq. (3) for a nontrivial topological sector (i.e., very large $\nu$ ) is delicate at best because, in addition, standard numerical implementations of the Bessel function can underflow for large $\nu$ [48], in which case the posterior evaluation of the determinant with Gaussian elimination might also be problematic.

This, of course, has the same implications for Eq. (2), where summing over all topological sectors is involved. Note also that it is possible to further characterize Eq. (2) analytically by plugging the integral representation Eq. (4) into Eq. (2), as was done in Ref. [49]. However, the resulting expression, as expected, loses its random matrix or determinantal form and it involves the evaluation of Bessel functions and a posterior multivariable integration with the same weight as in Eq. (4), but with the Bessel functions in the integrand [49]. Only in the two simplest cases, corresponding to one and two flavors (one and two spins flipped, in our forthcoming picture), does the partition function have an explicit analytic expression [49]. 
The connection made in this paper [Eq. (13)] opens the possibility of using numerical methods developed in the study of spin chains, such as White's density matrix renormalization group algorithm [50], or more concretely, some of its finite-temperature versions like Ref. [33], as an alternative method to compute the Leutwyler-Smilga integral for real $\beta$. For imaginary $\beta$, where classical simulation methods usually break down for large $\beta$, one may use quantum simulations with optical lattices. Indeed, as we comment below when discussing experimental implementations, the experiment [51] does exactly the job.

\section{1D XX MODEL AND THERMAL CORRELATION FUNCTIONS}

We now describe the result in Refs. [22-25] that relates some thermal correlation function of the XX model to a matrix model, which turns out to be the same as before.

We begin our discussion by presenting the spin chain model. The $S=1 / 2$ Heisenberg XX spin chain is one of the simplest integrable magnetic chains. It has a wellknown mapping, using the Jordan-Wigner transformation, to a free-fermion system [26]. This infinite chain (which we consider with periodic boundary conditions) is characterized by the Hamiltonian

$$
\hat{H}=-\frac{1}{2} \sum_{i} \sigma_{i}^{-} \otimes \sigma_{i+1}^{+}+\sigma_{i}^{-} \otimes \sigma_{i-1}^{+}+\frac{h}{2} \sum_{i}\left(\sigma_{i}^{z}-\mathbb{1}\right),
$$

where the summation is over all lattice sites and $h>0$. As usual, $\sigma_{i}^{ \pm}=\left(\sigma_{i}^{x} \pm i \sigma_{i}^{y}\right) / 2$, where $\sigma_{i}^{x}$ and $\sigma_{i}^{y}$ together with $\sigma_{i}^{z}$ denote the Pauli spin operators and $h$ represents the strength of an external magnetic field. The commutation relations are

$$
\left[\sigma_{i}^{+}, \sigma_{k}^{-}\right]=\sigma_{i}^{z} \delta_{i k}, \quad\left[\sigma_{i}^{z}, \sigma_{k}^{ \pm}\right]= \pm 2 \sigma_{i}^{ \pm} \delta_{i k} .
$$

These operators are nilpotent $\left(\sigma_{i}^{ \pm}\right)^{2}=0$, a property that will lead to a determinantal form for the correlation functions that we focus on. The other operator satisfies $\left(\sigma_{i}^{z}\right)^{2}=1$.

We begin by defining and describing the correlation functions of the model. Thermal correlation functions of spin chains have been studied for some time [52] and are known to admit determinantal expressions that are simpler in the case of the XX model (5) and have been studied explicitly more recently [22-25]. Following Ref. [25], the correlation function will be defined on a ferromagnetic state, which is characterized by having all the spins up $|\Uparrow\rangle=\bigotimes_{i}|\uparrow\rangle_{i}$, which satisfies $\sigma_{k}^{+}|\Uparrow\rangle=0$ for all $k$, and the state is also normalized $\langle\Uparrow \mid \Uparrow\rangle=1$. This state is annihilated by the Hamiltonian $\hat{H}|\Uparrow\rangle=0$ and the thermal correlation functions are defined by

$$
F_{j_{1}, \ldots, j_{K} ; l_{1}, \ldots, l_{K}}(\beta)=\left\langle\Uparrow\left|\sigma_{j_{1}}^{+} \cdots \sigma_{j_{K}}^{+} e^{-\beta \hat{H}} \sigma_{l_{1}}^{-} \cdots \sigma_{l_{K}}^{-}\right| \Uparrow\right\rangle .
$$

We consider first the particular case where we have only one spin down $K=1$,

$$
F_{j l}(\beta)=\left\langle\Uparrow\left|\sigma_{j}^{+} e^{-\beta \hat{H}} \sigma_{l}^{-}\right| \Uparrow\right\rangle
$$

By taking into account the commutation relation

$$
\begin{aligned}
{\left[\sigma_{j}^{+}, \hat{H}\right] } & =-\frac{1}{2} \sum_{k} \Lambda_{j k} \sigma_{j}^{z} \sigma_{k}^{+}-h \sum_{k} \sigma_{k}^{+} \delta_{j, k} \\
& =-\frac{1}{2} \sigma_{j}^{z}\left(\sigma_{j-1}^{+}+\sigma_{j+1}^{+}\right)-h \sigma_{j}^{+},
\end{aligned}
$$

where $\Lambda_{j k}=\delta_{j, k+1}+\delta_{j, k-1}$, together with the property $\langle\Uparrow| \sigma_{j}^{z}=\langle\Uparrow|$, it follows immediately [25]

$$
\begin{aligned}
\frac{d}{d \beta} F_{j l}(\beta) & =-\left\langle\Uparrow\left|\sigma_{j}^{+} \hat{H} e^{-\beta \hat{H}} \sigma_{l}^{-}\right| \Uparrow\right\rangle \\
& =\frac{1}{2}\left\langle\Uparrow\left|\left(\sigma_{j-1}^{+}+\sigma_{j+1}^{+}+2 h \sigma_{j}^{+}\right) e^{-\beta \hat{H}} \sigma_{l}^{-}\right| \Uparrow\right\rangle
\end{aligned}
$$

Hence,

$$
\frac{d}{d \beta} F_{j l}(\beta)=\frac{1}{2}\left[F_{j+1, l}(\beta)+F_{j-1, l}(\beta)\right]+h F_{j, l}(\beta) .
$$

This equation is that of a symmetric random walk on a line. We also remark that by commuting $\hat{H}$ with $\sigma_{l}^{-}$, there is an analogous difference equation but for subscript $l$ with fixed subscript $j$ [25]. Both equations are subject to the initial condition $F_{j l}(0)=\delta_{j l}$ and to boundary conditions that depend on the type of lattice considered. The results in Ref. [25] show that the case of general $K>1$ generalizes in a straightforward way and the multidimensional analog of Eq. (8) is obtained. The initial condition is the same, $F_{j_{1}, \ldots, j_{K} ; l_{1}, \ldots, l_{K}}(0)=\delta_{j_{1} l_{1}} \cdots \delta_{j_{K} l_{K}}$, and the correlation function also satisfies the conditions $F_{j_{1}, \ldots, j_{K} ; l_{1}, \ldots, l_{K}}(\beta)=0$, if $l_{r}=l_{s}$ or $j_{r}=j_{s}(r, s=1, \ldots, K)$, due to the nilpotency of the spin operators $\left(\sigma_{i}^{ \pm}\right)^{2}=0$. This "nonintersecting" property suggests a determinantal structure, and, indeed, the solution of the equation for general $K$ can be expressed as [25]

$$
F_{j_{1}, \ldots, j_{K} ; l_{1}, \ldots, l_{K}}(\beta)=\operatorname{det}_{1 \leq r, s \leq K}\left\{F_{j_{r} l_{s}}(\beta)\right\}
$$

where $F_{j l}(\beta)$ are the one-particle correlation functions satisfying Eq. (8). A matrix model expression for this determinant is given by $[25,53,54]$ 


$$
\begin{aligned}
& F_{j_{1}, \ldots, j_{K} ; l_{1}, \ldots, l_{K}}(f, \beta) \\
& =\frac{1}{(2 \pi)^{K} K !} \int_{-\pi}^{\pi} d \varphi_{1} \ldots \int_{-\pi}^{\pi} d \varphi_{K} \prod_{1 \leq j<k \leq K}\left|e^{i \varphi_{k}}-e^{i \varphi_{j}}\right|^{2} \\
& \quad \times\left(\prod_{j=1}^{K} f\left(\varphi_{j}\right)\right) \overline{\hat{s}_{\alpha}\left(e^{i \varphi_{1}}, \ldots, e^{i \varphi_{K}}\right)} \hat{s}_{\gamma}\left(e^{i \varphi_{1}}, \ldots, e^{i \varphi_{K}}\right),
\end{aligned}
$$

where $\hat{s}_{\lambda}\left(e^{i \varphi_{1}}, \ldots, e^{i \varphi_{n}}\right)$ is a Schur polynomial, a symmetric polynomial [55]. The relationship between the partitions $\alpha$ and $\gamma$ in the right-hand side of Eq. (10) and the $j$ and $l$ that appear in the thermal correlation function is [25]

$$
\alpha_{r}=j_{r}-K+r, \quad \gamma_{r}=l_{r}-K+r,
$$

and the weight function $f(\varphi)$ in the matrix model (10) is the generating function of the one-spin flip process (8). Therefore, noting that Eq. (10) is of the same form as Eq. (4) but more general due to the presence of two Schur polynomials, we can identify the number of flipped spins $K$ with the number of flavors $N_{f}$. In addition, $f(\varphi)$ being the generating function of the one-spin flip process (8) is generically given by

$$
f\left(\beta, e^{i \varphi}\right)=\sum_{j=-\infty}^{\infty} F_{j l}(\beta) e^{i \varphi j}
$$

and, in this particular case, by

$$
f\left(\beta, e^{i \varphi}\right)=f(0, \lambda) \exp \left\{\frac{\beta}{2}\left[2 h+\left(e^{i \varphi}+e^{-i \varphi}\right)\right]\right\} .
$$

Therefore, the weight function in Eq. (10) is

$$
f(\varphi)=e^{\beta(h+\cos \varphi)} .
$$

Notice that the analysis and the final result for the case of one flipped spin $K=1$ is identical to Glauber's seminal study of the kinetic Ising model [56]. In particular, the thermal average (6) behaves like the expectation of a single spin in an infinite ring in Ref. [56], with the time variable in Ref. [56] identified with our $\beta$.

Now, by considering the specific pattern of flipped spins $j_{r}=\nu+K-r$ and $l_{r}=K-r$, in Eq. (10) we get the partitions $\alpha=(0, \ldots, 0)$ and $\gamma=(\nu, \ldots, \nu)$. With these specific partitions, the respective Schur polynomials read [55],

$$
\hat{s}_{\alpha}\left(e^{i \varphi_{1}}, \ldots, e^{i \varphi_{K}}\right)=1, \hat{s}_{\gamma}\left(e^{i \varphi_{1}}, \ldots, e^{i \varphi_{K}}\right)=\prod_{j=1}^{K} e^{i \nu \varphi_{j}},
$$

and we recover exactly Eq. (4) from Eq. (10). That is, we obtain the key equation

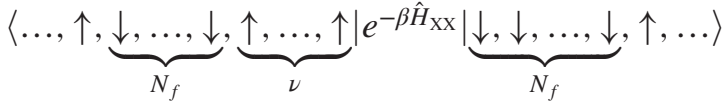

$$
\begin{aligned}
& =Z_{\nu, N_{f}}^{\mathrm{eff}}(m),
\end{aligned}
$$

where $\beta=V \Sigma m$.

\section{DICTIONARY QCD-SPIN CHAINS}

\section{A. Topological sector}

The first obvious connection arising from Eq. (13) is that the number of flavors $N_{f}$ corresponds to the number of spins down or, equivalently in the free fermion picture, the particle-number sector to which we restrict our attention in the $1 \mathrm{D}$ spin chain.

In addition, the shift $\nu$ in the positions of the spin-down particles at both sides of the thermal average in Eq. (13) induces a phase change in Eq. (4), which is responsible for the nontrivial topological sector of the QCD partition function. How do we understand this as some type of topological order present in the XX spin chain? The question is tricky since there is, in principle, no clear way to define topological order in 1D. A possible answer comes from the holographic principle, where one sees a (not necessarily normalized) 1D thermal state $e^{-\beta H}$ as the boundary of a $2 \mathrm{D}$ system. If the 2D system is topologically ordered, this should leave some signature in the 1D state. Starting with the seminal work of Li and Haldane [28], there have been several recent discussions about what this signature is [29-31,57]. Two key facts can be extracted from these works: (i) each topological sector in the bulk corresponds to projecting the thermal state of the boundary Hamiltonian in a different sector, and (ii) the bulk topology translates to some dynamical property on the boundary, and is hence related to the momentum. This agrees with the appearance of the translation operation $T$ in Eq. (13), which can be restated simply as

$$
\begin{aligned}
& Z_{\nu, N_{f}}^{\mathrm{eff}}(m) \\
& =\langle\ldots, \uparrow, \underbrace{\downarrow, \ldots, \downarrow}_{N_{f}}\left|e^{-\beta \hat{H}_{\mathrm{Xx}}} T^{-\nu}\right| \underbrace{\downarrow, \ldots, \downarrow}_{N_{f}}, \uparrow, \ldots\rangle \\
& =\langle\ldots, \uparrow, \underbrace{\downarrow, \ldots, \downarrow}_{N_{f}} \mid e^{-(\beta / 2) \hat{H}_{\mathrm{Xx}}} T^{-\nu} e^{-(\beta / 2) \hat{H}_{\mathrm{Xx}}} \underbrace{\downarrow, \ldots, \downarrow}_{N_{f}}, \uparrow, \ldots\rangle .
\end{aligned}
$$

The last equation very much resembles the momentum polarization tool introduced very recently in Ref. [57] as a way to detect nontrivial topological behavior [58].

To get (i) and (ii) and then show in a clearer way the topological content of $\nu$, it is better to go back to its Fourier dual parameter $\theta$. In order to avoid unnecessary mathematical complications, we now assume a finite chain of $2 L+1$ spins and define $\hat{T}=1 /(2 L+1) \sum_{\nu=-L}^{L} e^{i \nu \theta} T^{-\nu}$. By taking a basis of states $|k\rangle$ with definite momentum 
$T|k\rangle=e^{i[2 \pi k /(2 L+1)]}|k\rangle$ and changing variables $\theta=$ $\left(2 \pi \theta^{\prime}\right) /(2 L+1)$, one can see that

$$
\hat{T}|k\rangle=\frac{1}{2 L+1} \sum_{\nu=-L}^{L} e^{[2 \pi \nu i /(2 L+1)]\left(\theta^{\prime}-k\right)}|k\rangle=\delta_{k, \theta^{\prime}}|k\rangle,
$$

and, hence, $\hat{T}$ is just the projector $P_{\theta}$ onto the states with momentum $\theta$. Since it commutes trivially with the Hamiltonian, we finally get

$$
\begin{gathered}
\frac{1}{2 L+1} \sum_{\nu=-L}^{L} e^{i \nu \theta}\langle\ldots, \uparrow, \underbrace{\downarrow, \ldots, \downarrow}_{N_{f}}\left|e^{-\beta \hat{H}_{\mathrm{Xx}} T^{-\nu}}\right| \underbrace{\downarrow, \ldots, \downarrow}_{N_{f}}, \uparrow, \ldots\rangle \\
=\langle\ldots, \uparrow, \underbrace{\downarrow, \ldots, \downarrow}_{N_{f}}\left|P_{\theta} e^{-\beta \hat{H}_{\mathrm{Xx}}} P_{\theta}\right| \underbrace{\downarrow, \ldots, \downarrow}_{N_{f}}, \uparrow, \ldots\rangle .
\end{gathered}
$$

By considering the limit $L \rightarrow \infty$ and Eq. (2), this has the extra benefit of giving an interpretation of the global partition function $Z_{\mathrm{QCD}}^{\mathrm{eff}}(\theta)$ as a thermal average on the XX model when the Hamiltonian $\hat{H}$ is projected onto the sector of momentum $\theta$. That is,

$$
\begin{aligned}
& Z_{\mathrm{QCD}}^{\mathrm{eff}}(\theta) \\
& =\lim _{L \rightarrow \infty}(2 L+1)\langle\overbrace{\ldots, \uparrow, \underbrace{\downarrow, \ldots, \downarrow}_{N_{f}}}^{2 L+1}\left|P_{\theta} e^{-\beta \hat{H}_{\mathrm{Xx}}} P_{\theta}\right| \underbrace{\downarrow, \ldots, \downarrow}_{N_{f}}, \uparrow, \ldots\rangle .
\end{aligned}
$$

A mathematically fully rigorous argument of Eq. (14) is provided in the Appendix.

\section{B. Different matter content}

In the random matrix description of the thermal correlators, one can obtain symmetries other than the unitary symmetry of Eq. (10). As happens with the analogous setting of the Calogero model [59] and of nonintersecting random walks $[60,61]$, the inclusion of boundaries in the problem leads to other symmetries, such as orthogonal and symplectic symmetries. One of these cases is actually treated explicitly in Ref. [25], where an absorbing boundary condition at the origin is shown to lead to the same matrix model but with a correlation term between eigenvalues,

$$
\prod_{i=1}^{K} \sin ^{2} \theta_{i} \prod_{1 \leq j<k \leq K} \sin ^{2}\left(\frac{\theta_{j}-\theta_{k}}{2}\right) \sin ^{2}\left(\frac{\theta_{j}+\theta_{k}}{2}\right),
$$

instead of the usual Vandermonde in Eq. (10). These other situations have a counterpart in the low-energy QCD. In the chiral limit (with the masses of the fermions $m_{f} \rightarrow 0$ ), the relevant random matrix ensembles are the chiral Gaussian unitary, chiral Gaussian orthogonal, and chiral Gaussian symplectic ensembles [62], which are the ensembles that appear when the gauge theory has $S U\left(N_{c}\right)$ symmetry, with $N_{c} \geq 3$, for $S U(2)$ gauge group, and again for $S U\left(N_{c}\right)$ and $N_{c} \geq 3$, but in the adjoint representation (and fermions in the adjoint representation are Majorana fermions [21]), respectively [62]. These are precisely the resulting ensembles that describe the spin chain in the limit $\beta \rightarrow \infty$, because the weak-coupling limit of the Gross-Witten model is a Gaussian unitary ensemble [63]. The limitation in this case is due to the fact that $\beta$ is the parameter in the weight function of the resulting Gaussian ensemble, and, therefore, taking into account the identification of parameters in [62], this implies that there is a corresponding vanishing limit of the quark condensate. Thus, the correspondence in this setting is more subtle, due to the role played by Gaussian ensembles, which only emerge in our setting in the limit $\beta \rightarrow \infty$. Hence, these other cases have to be considered in more detail, but the point is that other relevant symmetries can be, in principle, described by considering boundaries in the spin chain model.

\section{Effects of a lattice}

In addition to other symmetries, obtained with the inclusion of boundaries in the spin chain, one can also consider additional interactions between neighboring spins in the chain. These new interactions modify the weight function in the matrix model (10) accordingly. This allows us to extend the correspondence between the spin chain and low-energy QCD to the case where the gauge theory is studied on the lattice $[64,65]$. The lattice breaks the chiral symmetry explicitly, and hence, the effects of the lattice spacing lead to new terms in chiral perturbation theory. This extended low-energy theory is known as Wilson chiral perturbation theory and leads to an extension of the matrix model (3), characterized by the addition of potential terms $[64,65]$

$$
\begin{aligned}
V(U)= & -a^{2} V W_{6}\left[\operatorname{Tr}\left(U+U^{\dagger}\right)\right]^{2}-a^{2} V W_{7}\left[\operatorname{Tr}\left(U-U^{\dagger}\right)\right]^{2} \\
& -a^{2} V W_{8} \operatorname{Tr}\left(U^{2}+U^{\dagger 2}\right)
\end{aligned}
$$

where $a$ denotes the lattice spacing and $W_{6}, W_{7}$, and $W_{8}$ are the new low-energy constants. The first two terms in Eq. (16) are multitrace potentials, which are more difficult to treat in general and, for the moment, have no known spin chain representation. However, these terms are expected to be suppressed in the large $N_{c}$ limit and are often not considered $[64,65]$. Interestingly enough, the remaining potential term in Eq. (16) can be described in the same manner as above, just by generalizing the spin chain to include next-to-nearest neighbor interactions. The resulting Hamiltonian is then 


$$
\begin{aligned}
\hat{H}= & -\frac{1}{2} \sum_{i} J_{1}\left(\sigma_{i}^{-} \otimes \sigma_{i+1}^{+}+\sigma_{i}^{-} \otimes \sigma_{i-1}^{+}\right) \\
& +J_{2}\left(\sigma_{i}^{-} \otimes \sigma_{i+2}^{+}+\sigma_{i}^{-} \otimes \sigma_{i-2}^{+}\right) .
\end{aligned}
$$

Note that we previously identified the $\beta$ parameter of the spin chain with a single combination of parameters of the effective field theory: $\beta=m V \Sigma$. Now, we have to identify $\beta J_{1}=m V \Sigma$ and $\beta J_{2}=2 a^{2} V W_{8}$. Thus, the relative strength of the interactions at first and second neighbors depends on the quotient between the masses of the quarks and the lattice spacing, together with the respective low-energy constants:

$$
\frac{J_{1}}{J_{2}}=\frac{m \Sigma}{2 a^{2} W_{8}} .
$$

\section{FINITE CHAIN ERRORS: EXPERIMENTAL ACCESSIBILITY}

It is also shown in Ref. [24], with a similar argument, that in the case of a finite chain of $L$ sites, the thermal average in the right-hand side of Eq. (13) is just the Riemann sum associated with the integral (4) when we evaluate on the vertices of a lattice division of the hypercube $[-\pi, \pi]^{N_{f}}$ of length $2 \pi / L$. A recent result by Baik and Liu [66] shows that the error obtained by this particular Riemann sum approximation decreases exponentially with $L$. More concretely, the relative error is $O\left(e^{-c\left(L-N_{f}\right)}\right)$ as $L-N_{f} \rightarrow \infty$, even if $N_{f}$ also goes to $\infty$.

This opens the door to a possible experimental measure of the quantity $Z_{\nu, N_{f}}^{\text {eff }}(\beta)$ as long as the experimental setup allows the following four steps: (1) implement the $\mathrm{XX}$ Hamiltonian $\sum_{i} \sigma_{i}^{+} \otimes \sigma_{i+1}^{-}+$H.c. (preferably with a magnetic field in the $z$ direction), (2) enforce the sector of $N_{f}$ particles, (3) stabilize the thermal state within the sector, and (4) measure the positions where the particles are. The crucial point is to realize that then the size of the chain, and the number of times that the experiment has to be done in order to approximate $Z_{\nu, N_{f}}^{\text {eff }}(\beta)$ within a relative error $\epsilon$, scales only polynomially with $\log (1 / \epsilon)$ (we treat $N_{f}$ and $\beta$ as constants). In order to see that, we consider a magnetic field $h \leq-2$ such that $|\Uparrow\rangle$ is the ground state. Note that, by Eq. (12), the magnetic field gives only a factor $e^{\beta h N_{f}}$ in the thermal average, so we can choose its value to our convenience. By the bound on the relative error, the length of the chain needs to scale only linearly with $\log (1 / \epsilon)$. The first step is to restrict to the sector given by $N_{f}$ spins down (particles in the free-fermion picture). In this way, one gets a Hilbert space $\mathcal{H}_{N_{f}}$, whose dimension scales polynomially with $L$ and, hence, with $\log (1 / \epsilon)$. The next step is to stabilize the system at the desired temperature, obtaining the thermal state $\rho_{\beta}$, which is just $e^{-\beta \tilde{H}} /\left(\operatorname{tr} e^{-\beta \tilde{H}}\right)$, with $\tilde{H}$ the restriction of $\hat{H}$ to $\mathcal{H}_{N_{f}}$. Since we have chosen the magnetic field for the state $|\Uparrow\rangle$ (the vacuum in the freefermion picture) to be the ground state, it is not difficult to see that $\operatorname{tr} e^{-\beta \tilde{H}} \leq \operatorname{dim} \mathcal{H}_{N_{f}}$, which makes

$$
\left|\left\langle\Uparrow\left|\sigma_{1}^{+} \cdots \sigma_{N_{f}}^{+} \rho_{\beta} \sigma_{N_{f}}^{-} \cdots \sigma_{1}^{-}\right| \Uparrow\right\rangle\right| \geq \frac{1}{\operatorname{polylog}\left(\frac{1}{\epsilon}\right)} .
$$

But the left-hand side of Eq. (17) is the probability of, given the state $\rho_{\beta}$ and measuring where the three particles are, obtaining that they are in positions one to three. Since this is larger than $1 /(\operatorname{polylog}(1 / \epsilon))$, the number of times one needs to make the experiment in order to get this value accurately scales also polynomially with $\log (1 / \epsilon)$.

It seems that ultracold gases in optical lattices are currently the best system to get the required steps (1)-(4). Indeed, very recently [51], the quantity $Z_{\nu, N_{f}}^{\text {eff }}(\beta)$ has been measured for chains of around 20 sites with imaginary $\beta$. The case of real $\beta$ does not seem completely out of reach. We briefly discuss why. There are two ways of getting the XX Hamiltonian in an optical lattice. One is to implement a 1D lattice hard-core boson Hamiltonian, which, by considering the particle-hole degree of freedom, is exactly the XX Hamiltonian. This (with an extra periodic confining potential) was already shown experimentally in Ref. [67], getting an array of 1D systems with a probability greater than $1 /\left(11 M^{1 / 3}\right)$ of having at least one with $M$ particles (for $M$ small). A different route to get such a Hamiltonian, proposed in Ref. [68] and experimentally obtained in Ref. [51], is to consider atoms with a spin degree of freedom in the insulating phase. By appropriately tuning the parameters, in second-order perturbation theory one obtains the XX Hamiltonian as the effective Hamiltonian of the system, though in a much smaller energy scale. By using the single spin addressing recently developed in Ref. [69] as done in Ref. [51], one can enforce the sector of a fixed number of particles. By the recent technique of high-resolution fluorescence imaging [70,71], one may also measure the position of the particles. The most subtle issue is stabilizing the thermal state. Indeed, the understanding of the thermodynamical properties of ultracold gases in optical lattices is a hot topic nowadays [72], which may lead to a solution of this problem in the near future. There are at least two possible routes for that [72]. One may start with a Bose-Einstein condensate in thermal equilibrium that is then adiabatically loaded into the lattice potential. Even though the XX Hamiltonian is integrable and, as shown, for instance, in Refs. [73,74], thermalization without an external bath is not guaranteed, the measures made in Ref. [67] are in excellent agreement with having a thermal state $[67,75]$. A second approach is to immerse the system in a reservoir with particles of a different species [72], so that we keep the number of particles constant in the lattice. Though there is no full study of the expected thermalization time, the recent estimate of Ref. [76] for the 
gap of the Davies Liouvillian-the one modeling the convergence to the thermal state of a system weakly coupled to a thermal bath-in the case of a fermion hoping on a line, allows one to be optimistic in this direction.

\section{THIRD-ORDER PHASE TRANSITION ON THE XX CHAIN AND THE TRACY-WIDOM LAW}

The final implication of Eq. (13) is the existence of a third-order phase transition hidden in the XX model [77] - the so-called Gross-Witten transition. Recall here that, as was shown in the seminal paper [32], if we consider the t' Hooft parameter $\lambda=K / \beta$, and we make $K \rightarrow \infty$ while keeping $\lambda$ constant, we obtain a double-scaling limit in $Z_{\nu=0, K}(\beta / V \Sigma)$-now we call it $Z_{\mathrm{GW}}(\beta, U(K))$ since it is the partition function of the Gross-Witten model with gauge group $U(K)$-with a third-order phase transition between the two regimes. Formally, the limit for the free energy

$$
F_{K}(\lambda)=\frac{1}{K^{2}} \ln Z_{\mathrm{GW}}(\beta, U(K))
$$

gives us [32]

$$
\lim _{K \rightarrow \infty} F_{K}(\lambda)= \begin{cases}\frac{1}{4 \lambda^{2}} & \lambda \geq 1 \\ \frac{1}{\lambda}+\frac{1}{2} \ln \lambda-\frac{3}{4} & \lambda<1 .\end{cases}
$$

By Eq. (13), which now reads

$$
\begin{aligned}
& \langle\ldots, \uparrow, \underbrace{\downarrow, \ldots, \downarrow, \downarrow}_{K}\left|e^{-\beta \hat{H}_{\mathrm{Xx}}}\right| \underbrace{\downarrow, \downarrow, \ldots, \downarrow}_{K}, \uparrow, \ldots\rangle \\
& =Z_{\mathrm{GW}}(\beta, U(K)),
\end{aligned}
$$

this is a phase transition in the XX model. Notice that now the correspondence is between the number of flipped spins and the rank of the gauge group. It is noteworthy that the above-mentioned exponentially small error for chains of finite size $L$ also holds in the double-scaling limit $[66,78]$. In particular, for the two phases, it holds that, if $R(L, K, \beta)$ denotes the Riemann sum, which is the partition function of the finite size spin chain, divided by the multiple integral (the partition function of the infinite chain), then [78] $R(L, K, \beta)=1+O\left(e^{-c K}\right)$ if $L>(1+\epsilon) \mu(K, \beta)$ (with $\epsilon>0)$, where

$$
\mu(K, \beta):= \begin{cases}2 \sqrt{K \beta} & \lambda<1 \\ K+\beta & \lambda \geq 1\end{cases}
$$

Note the different scaling of the finite length $L$ in terms of the number of flipped spins and the inverse temperature, depending on the phase. This property is a direct consequence of the discreteness of the associated random matrix ensemble.
On the other hand, one may argue that this phase transition happens only in a very unnatural limit of the spin chain parameters. However, there are signatures of this phase transition (a crossover) for very small values of $K$, as can be seen from the plots for $K=1,2,3$ in Ref. [79]. Actually, stronger results are available, since the following estimate holds in the strong-coupling phase $(\lambda \geq 1)[80]$

$$
\left|F_{K}(\lambda)-F(\lambda)\right| \leq C e^{-c K},
$$

for some constants $C$ and $c$ and $F(\lambda)=\lim _{K \rightarrow \infty} F_{K}(\lambda)$. This predicts an exponentially small departure, in the strong-coupling phase, for the case of a finite number of flipped spins $K$.

Note that this result holds for the free energy, which is the logarithm of the thermal correlator, and not just the partition function. The other phase is a bit more delicate to analyze and it is known that the finite rank case has $1 / K$ and higher-order corrections [81]. The resummation of all of the infinitely many terms is, of course, a complex and delicate issue, but more recent results show that the departure with the infinite rank case also decays quickly in the weak-coupling phase [82].

Thus, taking into account the results on finite chain errors and the comments above on experimental accessibility, one may be able to observe the Gross-Witten phase transition experimentally in a spin chain.

In addition to the work of Gross and Witten, the random matrix ensemble (4) with $\nu=0$ is central in the groundbreaking description of the asymptotics of the length of the longest increasing subsequence in random permutations [80]. In Ref. [80], it was proved that

$$
K=\beta+x(\beta / 2)^{1 / 3}
$$

for $\beta \rightarrow \infty$, then

$$
e^{-\beta^{2} / 4} Z_{\mathrm{GW}}(\beta, U(K)) \rightarrow F_{2}(x),
$$

where $F_{2}(x)$ is the celebrated Tracy-Widom distribution [83]. which can be given in terms of the Fredholm determinant of an Airy kernel or through an integral representation involving a solution of the Painlevé II equation, from which asymptotic expansions for $F_{2}(x)$ follow Refs. [27,83]. Notice that the factor $e^{-\beta^{2} / 4}$ in Ref. (22) is the normalization constant of the matrix model in the limit $K \rightarrow \infty$. In this way, the left-hand side of Eq. (22) is actually

$$
F_{\{K-r\}_{r=0}^{K-1},\{K-r\}_{r=0}^{K-1}}(\beta) \text { with } \lim _{K \rightarrow \infty} F\left(\beta, h^{*}\right)=1 .
$$

If the spin chain does not have the magnetic field term in Eq. (5), then one has to add the prefactor in Eq. (22) by hand. With the magnetic field $h$, there is an overall prefactor $\exp (h \beta K)$ outside the matrix model integral 
representation, and hence, to observe the Tracy-Widom shape that emerges in the scaling region above, one can fine-tune the magnetic field to $h^{*}=(\beta / 4 K) \sim 1 / 4$.

Notice that the result above zooms in the $\lambda=1$ region where the third-order phase transition occurs, and it describes typical and small fluctuations of order $\mathcal{O}\left(K^{-2 / 3}\right)$ in the transition point $[84,85]$. The relevance of the Tracy-Widom distribution resides in its large universality, and the universal fluctuations that it characterizes have been measured in recent experiments studying the height distribution of interfaces, in particular, in the slow combustion of paper and in turbulent liquid crystals $[86,87]$. Note that it makes its appearance in 2D classical statistical mechanics systems [88], whereas we are proposing that a corresponding result also holds in a quantum 1D system, the Heisenberg XX model.

\section{CONCLUSIONS}

We uncover a connection between QCD and the XX model using random matrix models, which allows us to establish a dictionary between both worlds as sketched in Table I. This opens an avenue to connect different quantum field theories with 1D spin chain Hamiltonians. The case of Chern-Simons theory is especially interesting due to its connections with topology, knot theory, and the fractional quantum Hall effect. We will make a full study in a forthcoming paper, showing that Table I also applies, albeit with a different 1D spin Hamiltonian.

\section{ACKNOWLEDGMENTS}

We thank Gernot Akemann, Mari Carmen Bañuls, Ignacio Cirac, Daniel Fernández-Fraile, Juan José García-Ripoll, Jose Ignacio Latorre, Belén Paredes, Grégory Schehr, Germán Sierra, and Pere Talavera for many useful comments and suggestions. We are indebted to Jinho Baik for very precise explanations of the results in Ref. [66]. This work has been partially funded by Spanish Grants No. MTM2011-26912 and QUITEMAD, Spanish program Juan de la Cierva, and European CHIST-ERA project CQC.

\section{APPENDIX: PROOF OF EQ. (14)}

We use some simple Banach space tools and notations for the proof of Eq. (14). Recall that $\ell_{1}$ is the Banach space of sequences $x=\left(x_{n}\right)_{n \in \mathbb{Z}}$ such that $\|x\|_{1}=\sum_{n=-\infty}^{\infty}\left|x_{n}\right|<\infty$. It is clear that the direct sum (or Cartesian product) of a finite number of copies of $\ell_{1}, \oplus_{k=1}^{K} \ell_{1}$, can be seen as another $\ell_{1}$ just considering the norm $\left\|\left(x^{1}, \ldots, x^{K}\right)\right\|=$ $\sum_{k=1}^{K}\left\|x^{k}\right\|_{1}$. Given a sequence $\left(x^{k}\right)_{k \in \mathbb{N}} \subset \ell_{1}$, one says that it converges to $x \in \ell_{1}$ if $\left\|x^{k}-x\right\|_{1} \rightarrow 0$. In a direct sum as above, convergence is simply equivalent to convergence in each of the factors. We use the following characterization of convergence for positive sequences in $\ell_{1}$ : given a sequence $\left(x^{k}\right)_{k \in \mathbb{N}} \subset \ell_{1}$, such that $x_{n}^{k} \geq 0$ for all $k, n$, $\lim _{k} x_{n}^{k}=x_{n}$ for all $n$, and $\lim _{k} \sum_{n=-\infty}^{\infty} x_{n}^{k}=\sum_{n=-\infty}^{\infty} x_{n}$, then $x^{k}$ converges in norm to $x \in \ell_{1}$.

We consider $N_{f} \times N_{f}$ matrices with values in $\ell_{1}$. We identify each column of the matrix with $X_{1}=\bigoplus_{k=1}^{N_{f}} \ell_{1}$, which is another $\ell_{1}$. The pointwise determinant

$$
\operatorname{Det}_{1}: \underbrace{X_{1} \times \cdots \times X_{1}}_{N_{f}} \rightarrow \ell_{1}
$$

is a continuous multilinear map on $X_{1}$ with values on $\ell_{1}$.

We fix $\beta=V \Sigma m$ and consider $\nu$ as the variable (getting sequences in $\nu \in \mathbb{Z}$ ).

We denote

$R_{L}(\nu)=\chi_{[-L, L]}(\nu)\langle\overbrace{\ldots, \uparrow, \underbrace{\downarrow}_{N_{f}}, \ldots, \downarrow}^{2 L+1}\left|e^{-\beta \hat{H}_{\mathrm{Xx}}} T^{-\nu}\right| \underbrace{\downarrow, \ldots, \downarrow}_{N_{f}}, \uparrow, \ldots\rangle$

and

$$
R(\nu)=\lim _{L \rightarrow \infty} R_{L}(\nu)\left[=Z_{\nu}^{\mathrm{eff}}(m)\right] .
$$

From the results in Refs [21,24] [see also Eq. (9)], we know that both $R_{L}$ and $R$ are determinants of a Toeplitz matrix

$$
\begin{aligned}
R_{L}(\nu) & =\chi_{[-L, L]}(\nu)\left|\begin{array}{cccc}
q_{\nu}^{L}(\beta) & q_{\nu+1}^{L}(\beta) & \cdots & q_{\nu+K-1}^{L}(\beta) \\
q_{\nu-1}^{L}(\beta) & q_{\nu}^{L}(\beta) & \cdots & q_{\nu+K-2}^{L}(\beta) \\
\cdots & \cdots & \cdots & \cdots \\
q_{\nu-K+1}^{L}(\beta) & q_{\nu-K+2}^{L}(\beta) & \cdots & q_{\nu}^{L}(\beta)
\end{array}\right|, \\
R(\nu) & =\left|\begin{array}{cccc}
I_{\nu}(\beta) & I_{\nu+1}(\beta) & \cdots & I_{\nu+K-1}(\beta) \\
I_{\nu-1}(\beta) & I_{\nu}(\beta) & \cdots & I_{\nu+K-2}(\beta) \\
\cdots & \cdots & \cdots & \cdots \\
I_{\nu-K+1}(\beta) & I_{\nu-K+2}(\beta) & \cdots & I_{\nu}(\beta)
\end{array}\right|,
\end{aligned}
$$

where $I_{k}(\beta)$ is the Bessel function of the imaginary argument and

$$
q_{k}^{L}(\beta)=\frac{1}{2 L+1} \sum_{s=-L}^{L} e^{2 \pi i s k /(2 L+1)} e^{\beta \cos 2 \pi s /(2 L+1)},
$$

which is trivially periodic (in $k$ ) with period $2 L+1$. As noted in the finite chain analysis in the main text, the first expression is just a Riemann sum associated with the integral representation of the Bessel function $I_{k}(\beta)$, which shows that $\lim _{L} q_{k}^{L}(\beta)=I_{k}(\beta)$, for all $k \in \mathbb{Z}$. Moreover, $I_{k}(\beta) \geq 0, q_{k}^{L}(\beta) \geq 0$, and we have the equalities

$$
\sum_{k=-L}^{L} q_{k}^{L}(\beta)=e^{\beta}=\sum_{j=-\infty}^{\infty} I_{j}(\beta) .
$$


By the characterization given above, for any fixed $r \in \mathbb{Z}$, we get that $\left[\chi_{[-L, L]}(\nu) q_{r+\nu}^{L}(\beta)\right]_{L} \subset \ell_{1}$ converges to $\left[I_{r+\nu}(\beta)\right]_{\nu} \in \ell_{1}$. Now, using the continuity of $\operatorname{Det}_{1}$, we get that $R_{L}$ converges to $R$ on $\ell_{1}$. This implies trivially weak convergence; that is, for any bounded sequence $\left(y_{\nu}\right)_{\nu \in \mathbb{Z}}$,

$$
\lim _{L \rightarrow \infty} \sum_{\nu=-\infty}^{\infty} y_{\nu} R_{L}(\nu)=\sum_{\nu=-\infty}^{\infty} y_{\nu} R(\nu)
$$

By taking $y_{\nu}=e^{i \theta \nu}$, we get

$$
\begin{aligned}
& Z_{\mathrm{QCD}}^{\mathrm{eff}}(\theta)=\sum_{\nu=-\infty}^{\infty} e^{i \nu \theta} Z_{\nu}^{\mathrm{eff}}(m) \\
& =\lim _{L \rightarrow \infty} \sum_{\nu=-L}^{L} e^{i \nu \theta} R_{L}(\nu)
\end{aligned}
$$

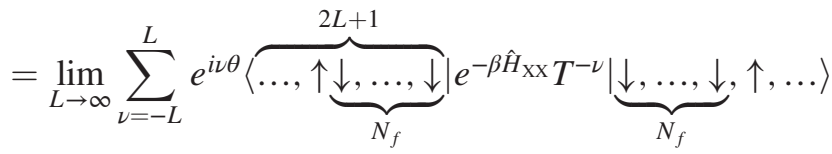

$$
\begin{aligned}
& =\lim _{L \rightarrow \infty}(2 L+1)\langle\overbrace{\ldots, \uparrow \underbrace{\downarrow}_{N_{f}} \underbrace{L+\ldots, \downarrow}}\left|P_{\theta} e^{-\beta \hat{H}_{\mathrm{xx}}} P_{\theta}\right| \underbrace{\downarrow, \ldots, \downarrow}_{N_{f}}, \uparrow, \ldots\rangle,
\end{aligned}
$$

which finishes the argument.

[1] H. D. Politzer, Reliable Perturbative Results for Strong Interactions?, Phys. Rev. Lett. 30, 1346 (1973); D. J. Gross and F. Wilczek, Ultraviolet Behavior of Non-Abelian Gauge Theories, Phys. Rev. Lett. 30, 1343 (1973).

[2] S. Weinberg, Phenomenological Lagrangians, Physica (Amsterdam) 96A, 327 (1979).

[3] J. Gasser and H. Leutwyler, Chiral Perturbation Theory to One Loop, Ann. Phys. (N.Y.) 158, 142 (1984); J. Gasser and H. Leutwyler, Chiral Perturbation Theory: Expansions in the Mass of the Strange Quark, Nucl. Phys. B250, 465 (1985).

[4] J. Gasser and H. Leutwyler, Thermodynamics of Chiral Symmetry, Phys. Lett. B 188, 477 (1987).

[5] K. Wilson, Confinement of Quarks, Phys. Rev. D 10, 2445 (1974).

[6] M. Creutz, Quarks, Gluons and Lattices (Cambridge University Press, Cambridge, England, 1985).

[7] J. Smit, Introduction to Quantum Fields on a Lattice (Cambridge University Press, Cambridge, England, 2002).

[8] S. J. Brodsky, H. C. Pauli, and S. S. Pinsky, Quantum Chromodynamics and Other Field Theories on the Light Cone, Phys. Rep. 301, 299 (1998).

[9] G. P. Korchemsky, Review of AdS/CFT Integrability, Chapter IV.4: Integrability in $Q C D$ and $\mathcal{N}<4$ SYM, Lett. Math. Phys. 99, 425 (2012).

[10] R. P. Feynman, Simulating Physics with Computers, Int. J. Theor. Phys. 21, 467 (1982).

[11] M. Lewenstein, A. Sanpera, V. Ahufingerb, B. Damskic, A. Sen(De), and U. Send, Ultracold Atomic Gases in Optical Lattices: Mimicking Condensed Matter Physics and Beyond, Adv. Phys. 56, 243 (2007).
[12] M. Lewenstein, A. Sanpera, and V. Ahufinger, Ultracold Atoms in Optical Lattices: Simulating Quantum Many-Body Systems (Oxford University Press, Oxford, 2012).

[13] S. P. Jordan, K. S. M. Lee, and J. Preskill, Quantum Algorithms for Quantum Field Theories, Science 336, 1130 (2012).

[14] S. P. Jordan, K. S. M. Lee, and J. Preskill, Quantum Computation of Scattering in Scalar Quantum Field Theories, arXiv:1112.4833.

[15] L. Tagliacozzo, A. Celia, A. Zamora, and M. Lewenstein, Optical Abelian Lattice Gauge Theories, Ann. Phys. (Amsterdam) 330, 160 (2013).

[16] L. Tagliacozzo, A. Celi, P. Orland, M. W. Mitchell, and M. Lewenstein, Simulations of Non-Abelian Gauge Theories with Optical Lattices, Nat. Commun. 4, 2615 (2013).

[17] D. Banerjee, M. Dalmonte, M. Müller, E. Rico, P. Stebler, U.-J. Wiese, and P. Zoller, Atomic Quantum Simulation of Dynamical Gauge Fields Coupled to Fermionic Matter: From String Breaking to Evolution after a Quench, Phys. Rev. Lett. 109, 175302 (2012).

[18] D. Banerjee, M. Bögli, M. Dalmonte, E. Rico, P. Stebler, U.-J. Wiese, and P. Zoller, Atomic Quantum Simulation of $U(N)$ and $S U(N)$ Non-Abelian Lattice Gauge Theories, Phys. Rev. Lett. 110, 125303 (2013).

[19] E. Zohar, J. I. Cirac, and B. Reznik, Simulating Compact Quantum Electrodynamics with Ultracold Atoms: Probing Confinement and Nonperturbative Effects, Phys. Rev. Lett. 109, 125302 (2012).

[20] E. Zohar, J. I. Cirac, and B. Reznik, Simulating $(2+1)$ Dimensional Lattice QED with Dynamical Matter Using Ultracold Atoms, Phys. Rev. Lett. 110, 055302 (2013); Cold-Atom Quantum Simulator for SU(2) Yang-Mills Lattice Gauge Theory, Phys. Rev. Lett. 110, 125304 (2013). 
[21] H. Leutwyler and A. Smilga, Spectrum of Dirac Operator and Role of Winding Number in $Q C D$, Phys. Rev. D 46, 5607 (1992).

[22] N. M. Bogoliubov, XXO Heisenberg Chain and Random Walks, J. Math. Sci. 138, 5636 (2006).

[23] N. M. Bogoliubov, Integrable Models for Vicious and Friendly Walkers, J. Math. Sci. 143, 2729 (2007).

[24] N. M. Bogoliubov and C. Malyshev, The Correlation Functions of the XXZ Heisenberg Chain in the Case of Zero or Infinite Anisotropy, and Random Walks of Vicious Walkers, St. Petersburg Math. J. 22, 359 (2011).

[25] N. M. Bogoliubov, A. G. Pronko, and J. Timonen, Scaling of Many-Particle Correlations in a Dissipative Sandpile, arXiv:1102.5639.

[26] E. Lieb, T. Schultz, and D. Mattis, Two Soluble Models of an Antiferromagnetic Chain, Ann. Phys. (N.Y.) 16, 407 (1961).

[27] P. J. Forrester, Log-Gases and Random Matrices (Princeton University Press, Princeton, NJ, 2010).

[28] H. Li and F. D. M. Haldane, Entanglement Spectrum as a Generalization of Entanglement Entropy: Identification of Topological Order in Non-Abelian Fractional Quantum Hall Effect States, Phys. Rev. Lett. 101, 010504 (2008).

[29] X. L. Qi, H. Katsura, and A. W. W. Ludwig, General Relationship between the Entanglement Spectrum and the Edge State Spectrum of Topological Quantum States, Phys. Rev. Lett. 108, 196402 (2012).

[30] J. I. Cirac, D. Poilblanc, N. Schuch, and F. Verstraete, Entanglement Spectrum and Boundary Theories with Projected Entangled-Pair States, Phys. Rev. B 83, 245134 (2011).

[31] N. Schuch, D. Poilblanc, J. I. Cirac, and D. Perez-Garcia, Topological Order in PEPS: Transfer Operator and Boundary Hamiltonians, Phys. Rev. Lett. 111, 090501 (2013).

[32] D. J. Gross and E. Witten, Possible Third-Order Phase Transition in the Large-N Lattice Gauge Theory, Phys. Rev. D 21, 446 (1980).

[33] F. Verstraete, J. J. Garcia-Ripoll, and J. I. Cirac, Matrix Product Density Operators: Simulation of Finite-Temperature and Dissipative Systems, Phys. Rev. Lett. 93, 207204 (2004).

[34] $m_{\pi}$ denotes the mass of the lightest Goldstone boson and can be expressed in terms of the low-energy constants $F, \Sigma$ and the masses of the quarks. See Ref. [21] or Ref. [35] for details.

[35] J. J. M. Verbaarschot, $Q C D$, Chiral Random Matrix Theory and Integrability, arXiv:hep-th/0502029.

[36] Y. Makeenko, Methods of Contemporary Gauge Theory (Cambridge University Press, Cambridge, England, 2002).

[37] M.Z. Hasan and C.L. Kane, Colloquium: Topological Insulators, Rev. Mod. Phys. 82, 3045 (2010).

[38] For $\nu \neq 0$, the matrix model describes the determinant of a Wilson loop [P. Rossi, On the Exact Evaluation of $\left\langle\operatorname{det} U_{p}\right\rangle$ in a Lattice Gauge Model, Phys. Lett. 117B, 72 (1982)] instead of the partition function of the 2D YangMills theory.

[39] O. Aharony, J. Marsano, S. Minwalla, K. Papadodimas, and M. Van Raamsdonk, The Hagedorn/Deconfinement Phase Transition in Weakly Coupled Large N Gauge Theories, Adv. Theor. Math. Phys. 8, 603 (2004).
[40] F. Bursa and M. Teper, Strong to Weak Coupling Transitions of $S U(N)$ Gauge Theories in $2+1$ Dimensions, Phys. Rev. D 74, 125010 (2006).

[41] L. N. Trefethen and J. A. C. Weideman, The Exponentially Convergent Trapezoidal Rule, SIAM Rev. (to be published)

[42] U.D. Jentschura and E Lötstedt, Numerical Calculation of Bessel, Hankel and Airy Functions, Comput. Phys. Commun. 183, 506 (2012).

[43] P. Debye, Näherungsformeln für die Zylinderfunktionen für Groe Werte des Arguments und Unbeschränkt Veränderliche Werte des Index, Math. Ann. 67, 535 (1909).

[44] F. W. J. Olver, Asymptotics and Special Functions (Academic Press, New York, 1974); reprinted as AKP Classics (A. K. Peters Ltd., Wellesley, MA, 1997).

[45] F. W. J. Olver, D. W. Lozier, R. F. Boisvert, and C. W. Clark, NIST Handbook of Mathematical Functions (Cambridge University Press, Cambridge, England, 2010).

[46] Resurgence makes reference to the fact that the coefficients $a_{n}$ of a Poincaré asymptotic series have the following special feature: the coefficients in the asymptotic expansions of $a_{n}$, as $n \rightarrow \infty$, are equal to themselves or related to the coefficients $a_{m}$.

[47] M. V. Berry, Uniform Asymptotic Smoothing of Stokes's Discontinuities, Proc. R. Soc. A 422, 7 (1989).

[48] D. E. Amos, Sandia Report No. 85-1018.

[49] J. Lenaghan and T. Wilke, Mesoscopic QCD and the Theta Vacua, Nucl. Phys. B624, 253 (2002).

[50] S. R. White, Density Matrix Formulation for Quantum Renormalization Groups, Phys. Rev. Lett. 69, 2863 (1992).

[51] T. Fukuhara, P. Schauss, M. Endres, S. Hild, M. Cheneau, I. Bloch, and C. Gross, Microscopic Observation of Magnon Bound States and Their Dynamics, Nature (London) 502, 76 (2013).

[52] F. Colomo, A. G. Izergin, V. E. Korepin, and V. Tognetti, Temperature Correlation Functions in the XXO Heisenberg Chain. I, Theor. Math. Phys. 94, 11 (1993).

[53] M. Adler and P. van Moerbeke, Virasoro Action on Schur Function Expansions, Skew Young Tableaux, and Random Walks, Commun. Pure Appl. Math. 58, 362 (2005).

[54] D. Bump and P. Diaconis, Toeplitz Minors, J. Comb. Theory Ser. A 97, 252 (2002).

[55] R. P. Stanley, Enumerative Combinatorics (Cambridge University Press, Cambridge, England, 2001), Vol. 2

[56] R. J. Glauber, Time-Dependent Statistics of the Ising Model, J. Math. Phys. (N.Y.) 4, 294 (1963).

[57] H-H. Tu, Y. Zhang, and X-L. Qi, Momentum Polarization: An Entanglement Measure of Topological Spin and Chiral Central Charge, Phys. Rev. B 88, 195412 (2013).

[58] Following Ref. [57], one can see how Eq. (13) relates directly to the generator $L_{0}$ of the Virasoro algebra associated with the CFT of the XX model. This emphasizes, in a way similar to Ref. [57], the topological content of $\nu$

[59] M. A. Olshanetsky and A. M. Perelomov, Classical Integrable Finite-Dimensional Systems Related to Lie Algebras, Phys. Rep. 71, 313 (1981).

[60] D. J. Grabiner, Brownian Motion in a Weyl Chamber, Non-Colliding Particles, and Random Matrices, Ann. Inst. H. Poincaré Probab. Statist. 35, 177 (1999).

[61] G. Schehr, S. N. Majumdar, A. Comtet, and P. J. Forrester, Reunion Probability of $\mathrm{N}$ Vicious Walkers: Typical and 
Large Fluctuations for Large N, J. Stat. Phys. 150, 491 (2013).

[62] J. Verbaarschot, Spectrum of the QCD Dirac Operator and Ciral Random Matrix Theory, Phys. Rev. Lett. 72, 2531 (1994).

[63] M. Romo and M. Tierz, Unitary Chern-Simons Matrix Model and the Villain Lattice Action, Phys. Rev. D 86, 045027 (2012).

[64] P. H. Damgaard, K. Splittorff, and J. J. M. Verbaarschot, Microscopic Spectrum of the Wilson Dirac Operator, Phys. Rev. Lett. 105, 162002 (2010).

[65] G. Akemann, P. H. Damgaard, K. Splittorff, and J. J. M. Verbaarschot, Spectrum of the Wilson Dirac Operator at Finite Lattice Spacings, Phys. Rev. D 83, 085014 (2011).

[66] J. Baik and Z. Liu, Discrete Toeplitz/Hankel Determinants and the Width of Nonintersecting Processes, Int. Math. Res. Not., doi: 10.1093/imrn/rnt143 (2013).

[67] B. Paredes, A. Widera, V. Murg, O. Mandel, S. Fölling, I. Cirac, G. V. Shlyapnikov, T. W. Hänsch, and I. Bloch, Tonks-Girardeau Gas of Ultracold Atoms in an Optical Lattice, Nature (London) 429, 277 (2004).

[68] L.-M. Duan, E. Demler, and M. D. Lukin, Controlling Spin Exchange Interactions of Ultracold Atoms in Optical Lattices, Phys. Rev. Lett. 91, 090402 (2003).

[69] C. Weitenberg, M. Endres, J. F. Sherson, M. Cheneau, P. Schauss, T. Fukuhara, I. Bloch, and S. Kuhr, Single-Spin Addressing in an Atomic Mott Insulator, Nature (London) 471, 319 (2011).

[70] W. S. Bakr, J. I. Gillen, A. Peng, S. Fölling, and M. Greiner, A Quantum Gas Microscope for Detecting Single Atoms in a Hubbard-Regime Optical Lattice, Nature (London) 462, 74 (2009).

[71] J. F. Sherson, C. Weitenberg, M. Endres, M. Cheneau, I. Bloch, and S. Kuhr, Single-Atom-Resolved Fluorescence Imaging of an Atomic Mott Insulator, Nature (London) 467, 68 (2010).

[72] D. C. McKay and B. DeMarco, Cooling in Strongly Correlated Optical Lattices: Prospects and Challenges, Rep. Prog. Phys. 74, 054401 (2011).

[73] T. Kinoshita, T. Wenger, and D.S. Weiss, A Quantum Newton's Cradle, Nature (London) 440, 900 (2006).
[74] M. Rigol, V. Dunjko, and M. Olshanii, Thermalization and Its Mechanism for Generic Isolated Quantum Systems, Nature (London) 452, 854 (2008).

[75] M. Rigol, Finite-Temperature Properties of Hard-Core Bosons Confined on One-Dimensional Optical Lattices, Phys. Rev. A 72, 063607 (2005).

[76] K. Temme, Lower Bounds to the Spectral Gap of Davies Generators, arXiv:1305.5591.

[77] Third-order phase transitions are rare in condensed matter systems. Another third-order phase transition was found recently in Ref. [89].

[78] J. Baik (private communication).

[79] C. B. Lang, P. Salomonson, and B. S. Skagerstam, A Study of Exactly Solvable Lattice Gauge Theories in Two Space-Time Dimensions, Phys. Lett. 100B, 29 (1981).

[80] J. Baik, P. Deift, and K. Johansson, On the Distribution of the Length of the Longest Increasing Subsequence of Random Permutations, J. Am. Math. Soc. 12, 1119 (1999).

[81] Y. Y. Goldschmidt, 1/n Expansion In Two-Dimensional Lattice Gauge Theory, J. Math. Phys. (N.Y.) 21, 1842 (1980).

[82] M. Marino, Nonperturbative Effects and Nonperturbative Definitions in Matrix Models and Topological Strings, J. High Energy Phys. 12 (2008) 114.

[83] C. A. Tracy and H. Widom, Level-Spacing Distributions and the Airy Kernel, Commun. Math. Phys. 159, 151 (1994).

[84] P. J. Forrester, S. N. Majumdar, and G. Schehr, Nonintersecting Brownian Walkers and Yang-Mills Theory on the Sphere, Nucl. Phys. B844, 500 (2011).

[85] Notice that we can write the condition (21) as $x=2^{1 / 3}\left(\lambda-1 / K^{2 / 3}\right)$, where $\lambda$ is the 't Hooft parameter.

[86] K. A. Takeuchi and M. Sano, Universal Fluctuations of Growing Interfaces: Evidence in Turbulent Liquid Crystals, Phys. Rev. Lett. 104, 230601 (2010).

[87] K. A. Takeuchi, M. Sano, T. Sasamoto, and H. Spohn, Growing Interfaces Uncover Universal Fluctuations Behind Scale Invariance, Sci. Rep. 1, 34 (2011).

[88] T. Kriecherbauer and J. Krug, A Pedestrian's View on Interacting Particle Systems, KPZ Universality and Random Matrices, J. Phys. A 43, 403001 (2010).

[89] S. Lerma H., S. M. A. Rombouts, J. Dukelsky, and G. Ortiz, Integrable Two-Channel $p_{x}+i p_{y}$-Wave Model of a Superfluid, Phys. Rev. B 84, 100503 (2011). 